\title{
The use of a modified critical plane model to assess multiaxial fatigue of steels with nonmetallic inclusions
}

Pedro Vinícius Sousa Machado ${ }^{1}$, Lucas Carneiro Araújo ${ }^{1}$, Marcos Venicius Soares ${ }^{2}$, and José Alexander Araújo ${ }^{1}$

${ }^{1}$ University of Brasília, Department of Mechanical Engineering, 70910-900 Brasília-DF, Brazil ${ }^{2}$ Catholic University of Rio de Janeiro, Department of Chemical and Material Engineering, 22453-901 Rio de Janeiro-RJ, Brazil

\begin{abstract}
The goal of this research is to investigate the detrimental effect of non-metallic inclusions on the fatigue strength of the AISI 4140 steel under multiaxial loading conditions. In order to do so, a multiaxial fatigue model based on the critical plane approach is coupled with Murakami's $\sqrt{ }$ area model. The proposed adaptation is very easy to calibrate and can also account for the higher probability of existing a fatal small defect as the volume of stresses material increases. Experimental multiaxial fatigue data were generated and compared with the estimates provided by the adapted multiaxial fatigue model and with its original version. The errors found are not higher than $10 \%$.
\end{abstract}

\section{Introduction}

The detrimental effect of nonmetallic inclusions on the fatigue strength of high strength steels has recently been the cause of costly failures in crankshafts of thermoelectric powerplants in Brazil. These steels are sensible to the presence of small defects such as cracks, scratches and nonmetallic inclusions, as fatigue cracks tend to initiate in these regions. In fact, nonmetallic inclusions are commonly seen in the fracture's fish eye on failed specimens made of AISI 4140 steel.

To tackle this problem, Murakami [1] introduced a model that relates the parameter $\sqrt{\text { area }}$ of a small artificial defect with a nominal fatigue limit under fully reversed uniaxial loading $\left(\sigma_{w}\right)$. Later, Yanase and Endo proposed a similar equation to predict the fatigue strength under fully reversed torsion $\left(\tau_{w}\right)$ [2]. Both models have been extended to estimate the fatigue limit of naturally defective materials, such as steels with nonmetallic inclusions, with the use of statistics of extreme value theory. Their approach not only allows an effortless and precise fatigue assessment, but it also permits the consideration of the size effect. This is crucial when sizable engineering components are designed, as the probability of a fatal small defect increases with the volume of material.

In this context, the Modified Wöhler Curve Method (MWCM) [3] will be coupled with the $\sqrt{\text { area }}$ parameter and include a statistical analysis so that it will be to deal with the multiaxial fatigue design of steels containing nonmetallic inclusions such as the AISI 4140 
steel. Some researchers $[2,4,5]$ have claimed that specific multiaxial criteria are required to design against fatigue for these type of defective materials. However, in this paper, it is show that it is possible to adapt classical critical plane approach to model these problems.

\section{Relationship between small defect's size and the fatigue limit}

Murakami [1] proposed the existence of a nominal fatigue limit $\left(\sigma_{w}\right.$ with $\left.R=-1\right)$ for a material with a small notch, according to Eq. 1. As reported by him, [6] once a crack nucleates around it, both crack and small defect should be treated as one entity. In the case of a small artificial hole, the square root of its projected area on the plane perpendicular to the maximum principal stress direction $(\sqrt{\text { area }})$ is used as a measure of the defect's size.

$$
\sigma_{w}=\frac{1.43(H v+120)}{(\sqrt{\text { area }})^{1 / 6}}
$$

where $H v$ is its hardness measured in Vickers. Eq. 1 already takes into account the defectcrack association.

Later, Yanese and Endo [4] proposed that the fatigue limit under fully reversed torsion could be calculated according to Eq. 2 .

$$
\tau_{w}=\frac{1.21(H v+120)}{(\sqrt{\text { area }})^{1 / 6}}
$$

As can be seen, the $\sigma_{w}$ and $\tau_{w}$ are trivial to compute if the $\sqrt{\text { area }}$ is known. Such is the case when artificially introduced defects are analysed. However, how about when nonmetallic inclusions of various shapes and sizes are the source of failures? In order to overcome this problem, Murakami invoked the method of statistics of extreme value [7] to estimate a very large $\sqrt{\text { area }}$ of an inclusion that is likely to be present in the analysed volume of material, designated $\sqrt{\text { area }}$ max . The inclusions' $\sqrt{\text { area }}$ follow a statistical distribution, hence determining $\sqrt{\text { area }}_{\text {max }}$ is relatively easy. Applying this theory is paramount for this problem, since fatigue cracks usually begin at very large inclusions in high strength steels such as the AISI 4140. Simply evaluating an average size of inclusions to rate the cleanliness of this material might lead to an overestimation of the fatigue limit. Therefore, once the $\sqrt{\text { area }}_{\text {max }}$ is known, the $\sigma_{w}$ and $\tau_{w}$ can be calculated according to Eq. 3 and Eq. 4.

$$
\begin{aligned}
\sigma_{w} & =\frac{1.41(H v+120)}{\left(\sqrt{\text { area }}_{\text {max }}\right)^{1 / 6}} \\
\tau_{w} & =\frac{1.19(H v+120)}{\left(\sqrt{\text { area }}_{\text {max }}\right)^{1 / 6}}
\end{aligned}
$$

Eq. 3 have shown errors of less than $10 \%$ compared to experiments for various materials as long as $\sqrt{\text { area }}_{\text {max }} \leq 1000 \mu m$ and $70 \leq H v \leq 700$.

\section{The Modified Wöhler Curve Method}

Susmel and Lazzarin [3] proposed the Modified Wöhler Curve Method (MWCM) to predict the fatigue damage of metallic materials under multiaxial loading conditions. They noted that cracks' initiation and early propagation happened in a critical material plane. It was defined as the plane experiencing the maximum amplitude of the shear stress, designated $\tau_{a}$, during a loading history. Following this initial stage, final propagation happens in the direction of maximum normal stress. The MWCM may be represented by the relation of Eq. 5 . 


$$
\lambda \geq \tau_{a}\left(\theta_{c}, \phi_{c}\right)+\kappa \frac{\sigma_{n, \max }}{\tau_{a}}\left(\theta_{c}, \phi_{c}\right)
$$

where $\lambda$ and $\kappa$ are material constants that can be obtained, according to Eq. 6, from fullyreversed push-pull (or rotating bending) $\left(\sigma_{0}\right)$ and fully reversed torsional $\left(\tau_{0}\right)$ fatigue tests. The term $\sigma_{n, \max }$ is the maximum normal stress on the critical plane. Eq. 5 indicates that the loads are safe, and failure is not expected before $2 \times 10^{6}$ cycles, as long as the right side of the inequation remains smaller than the material constant $\lambda$.

$$
\kappa=\tau_{0}-\frac{\sigma_{0}}{2} \quad \lambda=\tau_{0}
$$

The $\tau_{a}$ parameter will be computed by the Maximum Rectangular Hull (MRH) algorithm [8], which besides being very simple to implement, can take into account the effect of the non-proportional stress histories. In the MRH, the maximum shear stress amplitude is defined as half of the diagonal of the maximum rectangular hull that envelops the entire history of the shear stress vector component.

Susmel and co-workers also defined the variable $\rho$, given by Eq. 7, that represents the influence of the normal stress on the fatigue damage of the material.

$$
\rho=\frac{\sigma_{n, \max }}{\tau_{a}}
$$

However, if its influence is too great the model does not hold. More precisely, it should not be used if $\rho$ exceeds the value of $\rho_{\text {lim }}$, which is given by Eq. 8 .

$$
\rho_{\text {lim }}=\frac{\sigma_{0}}{2 \tau_{0}-\sigma_{0}}
$$

\section{Adapting the MWCM to materials containing small defects}

To extend the application of the MWCM criterion to estimate the limit of resistance of multiaxial fatigue in materials containing small defects, the following procedure is proposed:

i. Determine the hardness of the material and the $\sqrt{\text { area }}_{\text {max }}$ parameter;

ii. Estimate the uniaxial fatigue strength $\left(\sigma_{w}\right)$ according Eq. 3;

iii. Estimate the torsional fatigue strength $\left(\tau_{w}\right)$ according to Eq. 4 ;

iv. Determine material constants $\lambda$ and $\kappa$ from $\sigma_{w}$ and $\tau_{w}$ estimated in steps (ii) and (iii);

v. Assess the accuracy of the new adapted MWCM for defective materials considering the available data.

As opposed to Murakami's model, which assumes crack nucleation on the plane perpendicular to the direction of maximum principal stress, one of the premises of the MWCM is that crack nucleation occurs on planes experiencing the maximum amplitude of the shear stress.

\section{Experiments and material}

The material considered in this experimental program is the AISI 4140 steel oil quenched and tempered at $600{ }^{\circ} \mathrm{C}$ with mechanical properties reported in Table 1. Specimens were round with $\varnothing 10 \mathrm{~mm}$ in the test section and were designed according to an international standard [9].

Table 1. Mechanical properties of AISI 4140 steel.

\begin{tabular}{|c|c|c|c|}
\hline Yield Strength (MPa) & Tensile Strength (MPa) & Elongation (\%) & Vickers hardness \\
\hline 710 & 900 & 20 & 320 \\
\hline
\end{tabular}


A modified version of the staircase method was used to experimentally determine the material's fatigue limits $\sigma_{0}$ and $\tau_{0}$ [10]. In this method, the first specimen is subjected to a stress amplitude knowingly smaller than the expected fatigue limit. If $2 \times 10^{6}$ cycles are endured, one increment is added to the stress amplitude in the following test and the same specimen is reused. It goes on until the first specimen fails. The second specimen is then tested two increments below the amplitude of failure of the first specimen. The process is repeated until a satisfactory standard deviation is obtained.

On the other hand, $\sigma_{w}$ and $\tau_{w}$ of Eq. 3 and 4 need to be determined. In order to do so, a very large inclusion size $\left(\sqrt{\text { area }}_{\text {max }}\right)$ in a specimen must be estimated with statistics of extreme theory. This procedure is thoroughly explained by Murakami in some of his works [11]. Briefly, it consists of measuring the $\sqrt{\text { area }}$ of the largest inclusion, called $\sqrt{\text { area }}$ max,j, of each of several control regions of a representative sample of the material. The measurements are made on a polished surface perpendicular to the direction of the maximum principal stress. Then, plot the $\sqrt{\text { area }}_{\text {max, }}$ against the reduced variable $y_{j}$ given by Eq. 9 .

$$
y_{i}=-\ln \left[-\ln \left(\frac{j}{n+1}\right)\right]
$$

where $j$ is the inclusion number from a list in ascending order of $\sqrt{\operatorname{are}}_{\text {max }, j}$, and $n$ is the total number of inspected inclusions. If the resulting plot is approximately a straight line, it means that these extreme inclusions follow the Gumbel distribution. Therefore, it is possible to determine the $\sqrt{\text { area }}_{\text {max }}$ expected in the analysed prediction volume of material by defining a return period.

In the case of the fatigue test specimens, the prediction volume corresponds to its test section which is over $3 \times 10^{8}$ times smaller than a crankshaft of a thermoelectric powerplant. This amounts to a much higher probability of very large inclusions occurrences. It is one reason why fatigue limits determined in laboratories must be corrected when used to design sizable engineering components. To better understand this difference, the $\sqrt{\text { area }}$ max will be estimated for two prediction volumes: a specimen's test section and for a crankshaft.

Moreover, the analysis was made in two different samples of the material cut into two different planes. One at $90^{\circ}$ and the other at $45^{\circ}$, both relative to the longitudinal axis of the specimen. Each represent the planes perpendicular to the principal direction under fully reversed uniaxial and torsional loads respectively.

The multiaxial fatigue tests were conducted in the laboratories of the Department of Mechanical Engineering in the University of Brasilia. Such tests were carried out in two MTS $809 \mathrm{axial} /$ torsional Test Systems (100 $\mathrm{kN}$ and $1100 \mathrm{Nm}$ load capacity). Figure 1 depicts a broken specimen still mounted between the upper and lower grips. During the tests, temperature was kept in $20^{\circ} \mathrm{C}$ and the frequencies were maintained between 5 and $6 \mathrm{~Hz}$ depending on the load. Loading ratios $(\tau / \sigma)$ of $1,0.5$ and $\infty$ were considered. 


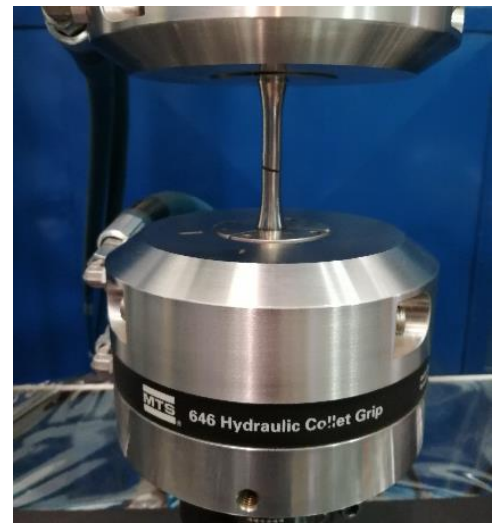

Fig. 1. Photo of broken specimen mounted in the MTS 809 axial/torsional test system.

\section{Results}

The results of $\sigma_{0}$ and $\tau_{0}$ obtained from the modified staircase method can be seen in Table 2 . They are corrected for a confidence interval of $90 \%$ and will be used to calibrate the MWCM as originally proposed.

Table 2. Fatigue limits of AISI 4140 steel determined experimentally

\begin{tabular}{|c|c|c|}
\hline $\left.\boldsymbol{\sigma}_{\mathbf{0}} \mathbf{( M P a}\right)$ & $\left.\boldsymbol{\tau}_{\mathbf{0}} \mathbf{( M P a}\right)$ & Confidence interval \\
\hline $375 \pm 20$ & $282 \pm 13$ & $90 \%$ \\
\hline
\end{tabular}

Values of $\sqrt{\text { area }}$ max on material planes at $90^{\circ}$ and $45^{\circ}$ in relation to the longitudinal axis of the specimens were estimated. Then the fatigue limits under uniaxial and torsional fully reversed loads, $\sigma_{w}$ and $\tau_{w}$, can be extracted from Eq. 3 and 4 . Also, it is possible to estimate an inclusion size for both prediction volumes, hence their corresponding fatigue limits. See the difference between the results of this statistical analysis in Table 3.

Table 3. Results of statistics of extreme value theory applied to inclusion's $\sqrt{\text { area }}$.

\begin{tabular}{|c|c|c|c|c|c|c|}
\hline $\begin{array}{c}\text { Prediction } \\
\text { Volume }\end{array}$ & $\begin{array}{c}\text { Volume } \\
\left(\mathbf{m m}^{\mathbf{3}}\right)\end{array}$ & $\begin{array}{c}\boldsymbol{\sigma}_{\boldsymbol{w}} \\
(\mathbf{M P a})\end{array}$ & $\begin{array}{c}\boldsymbol{\tau}_{\boldsymbol{w}} \\
(\mathbf{M P a})\end{array}$ & $\begin{array}{c}\sqrt{\boldsymbol{a r e a}}_{\text {max }} \\
(\boldsymbol{\mu m}) \text { at } \\
\mathbf{9 0}^{\circ}\end{array}$ & $\begin{array}{c}\sqrt{\boldsymbol{a r e a}}_{\text {max }} \\
(\boldsymbol{\mu m}) \text { at } \\
\mathbf{4 5}^{\circ}\end{array}$ & $\begin{array}{c}\text { Cumulative } \\
\text { distribution } \\
(\mathbf{\%})\end{array}$ \\
\hline 1 Specimen & $2.4 \times 10^{3}$ & 360 & 301 & 18.5 & 27.6 & 99.893 \\
\hline 2 Crankshaft & $7.9 \times 10^{9}$ & 311 & 260 & 63.5 & 65.4 & 99.9999996 \\
\hline
\end{tabular}

Using the torsional and uniaxial fatigue limits of the material presented in Table 2 and Table 3, the MWCM criteria can now be calibrated according to Eq. 6. The results are presented in Table 4.

Table 4. MWCM material constants

\begin{tabular}{|l|c|c|}
\hline & $\boldsymbol{\kappa}$ & $\boldsymbol{\lambda}$ \\
\hline With $\sqrt{\text { area }}_{\text {max }}$ of prediction volume 1 & 121 & 301 \\
\hline With $\sqrt{\text { area }}_{\text {max }}$ of Prediction volume 2 & 105.5 & 261 \\
\hline With $\tau_{0}$ and $\sigma_{0}$ & 94.5 & 282 \\
\hline
\end{tabular}

The multiaxial fatigue results can now be compared to the adapted MWCM criteria for each prediction volume and its original counterpart in relation to the multiaxial fatigue experiments. The results are shown in Fig. 2. 

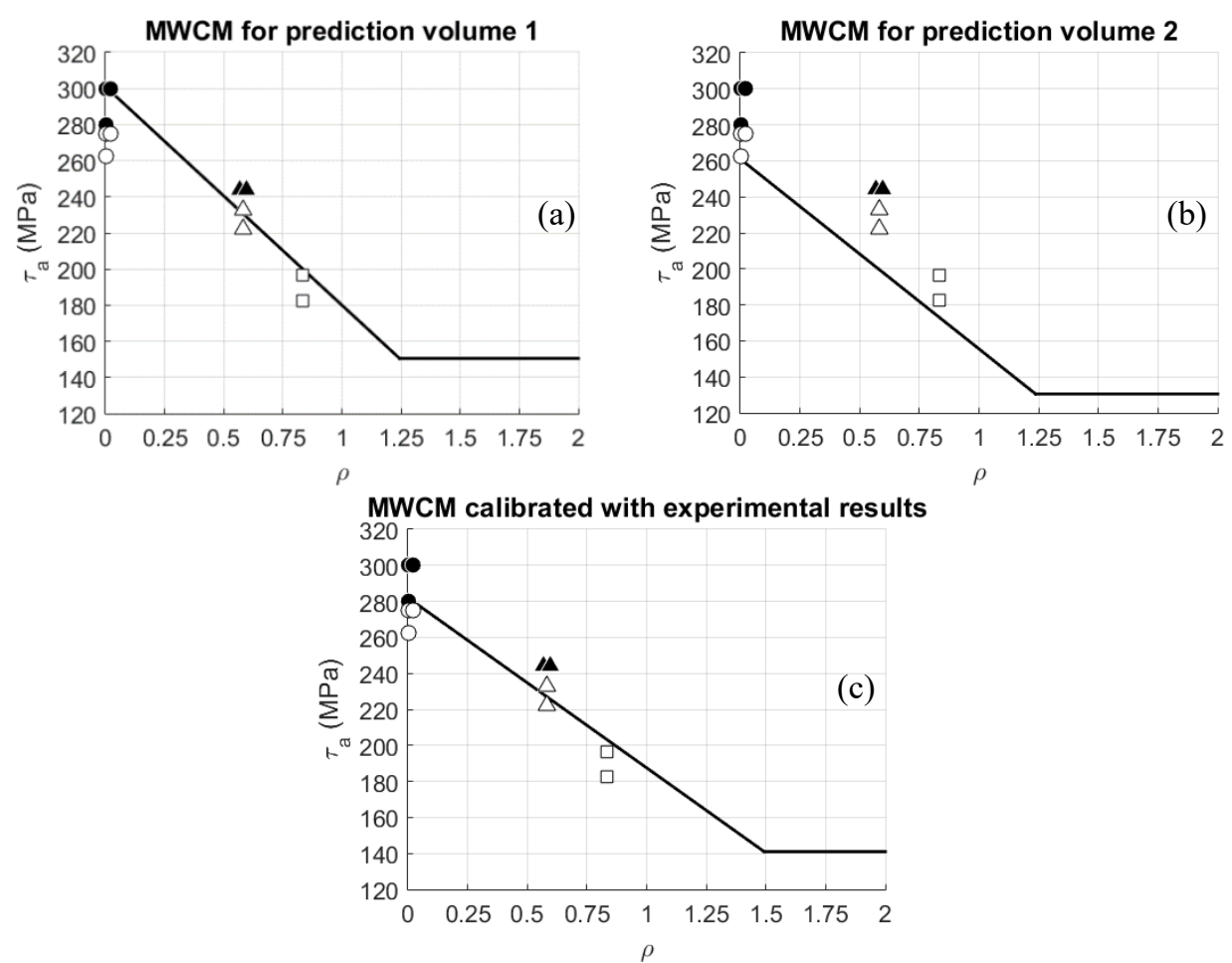

Fig. 2. (a) MWCM calibrated for the prediction volume of a specimen (b) MWCM calibrated for the prediction volume of a crankshaft of 5.9 tons (c) MWCM calibrated with $\tau_{0}$ and $\sigma_{0}$.

After each test, failed specimens were analysed on a scanning electron microscope. Inclusions were detected in the fracture's fish eye of some of them as can be seen in Fig. 3.

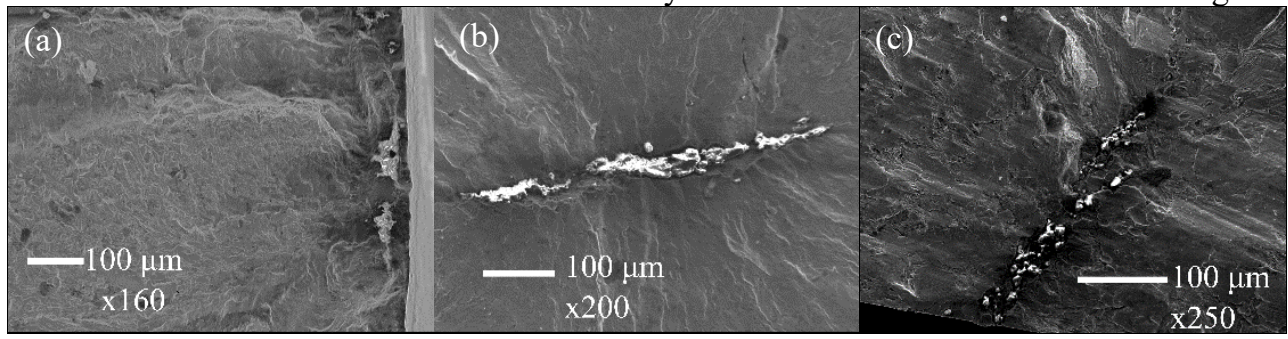

Fig. 3. Inclusions seen on fracture's fish eye of three specimens (a) specimen 1; (b) specimen 2; (c) specimen 3 .

Likewise, specimens that endured $2 \times 10^{6}$ cycles on a stress level just below the failure threshold were examined under an optical microscope. Non-propagated cracks were found beginning on the tip of surface inclusions, as shown in Fig. 4. 

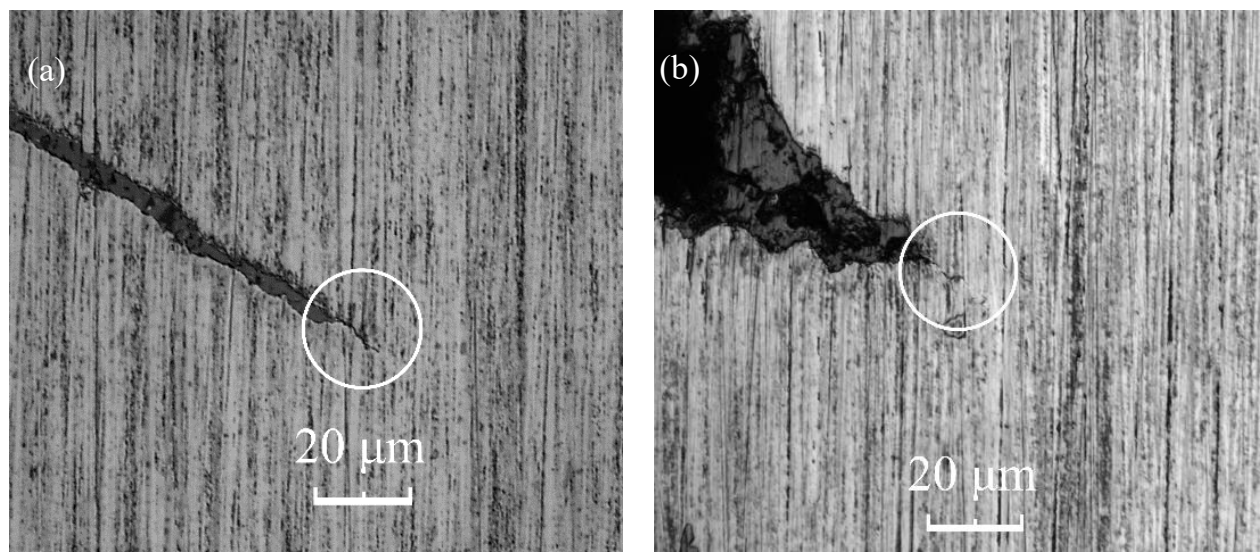

Fig. 4. Non-propagated cracks on surface inclusions (a) inclusion 1; (b) inclusion 2

The performance of the model may be assessed by Eq. 10. It compares the relative difference between the experimental result $\left(R_{E}\right)$ and the predicted result $\left(R_{P}\right)$, hence an Error Index $(E I)$ is extracted.

$$
E I=\frac{R_{E}-R_{P}}{R_{P}} \times 100 \%
$$

As the model used in the current paper is the MWCM, as presented previously, Eq. 10 becomes Eq. 11.

$$
E I=\frac{\tau_{a}\left(\theta_{c}, \phi_{c}\right)+\kappa \frac{\sigma_{n, \max }}{\tau_{a}}\left(\theta_{c}, \phi_{c}\right)-\lambda}{\lambda} \times 100 \%
$$

To compare the three approaches presented in Fig. 2, Eq. 11 is going to be applied to the experimental points in which the model failed to correctly predict the outcome, i.e.: failures bellow the diagram and runs-out above it. In this manner, a negative average error will indicate overall non-conservative predictions, while a positive average will indicate that the model has been conservative. The results are in Table 5.

Table 5. Performance assessment of the adapted MWCM.

\begin{tabular}{|c|c|c|c|}
\hline & $\begin{array}{c}\text { MWCM calibrated } \\
\text { from } \boldsymbol{\tau}_{\mathbf{0}} \text { and } \boldsymbol{\sigma}_{\mathbf{0}}\end{array}$ & $\begin{array}{c}\text { MWCM calibrate } \\
\text { from volume 1 }\end{array}$ & $\begin{array}{c}\text { MWCM calibrate } \\
\text { from volume 2 }\end{array}$ \\
\hline Error (\%) & $-0.7 \pm 1.4$ & $-1.7 \pm 3.1$ & $6.5 \pm 3.7$ \\
\hline
\end{tabular}

\section{Conclusions}

In this work an adapted version of the Modified Wöhler Curve Method [3] was proposed. The model was applied to evaluate the multiaxial fatigue limit of specimens made AISI 4140 steel containing nonmetallic inclusions. To adapt the model we introduced the $\sqrt{\text { area }}$ max parameter proposed by [1] to estimate the material constants $\kappa$ and $\lambda$ from the nominal fatigue limits of these steels under fully reversed uniaxial and torsional loading. The main advantage of Murakami's models is their capability of predicting the nominal fatigue strength of hard steels containing microscopic defects with relative ease, as estimating the parameter $\sqrt{\text { area }}_{\text {max }}$ of a steel is a simple process. In this context, it was shown that:

i. It is possible to determine good approximations of the material fatigue constants required by multiaxial criteria, such as the MWCM, without the need to run fatigue tests, which are lengthy and expensive. 
ii. Using statistics of extreme theory applied to nonmetallic inclusions, it is possible to consider the size effect in design of engineering components under multiaxial loading conditions. As the volume of stressed material increases the possibility of existing a larger fatal defect, which will reduce the fatigue limits, raises. The error results presented in Table 5 show that by means of the statistical analysis it is possible to easily obtain the constants for a more conservative MWCM criterion.

This research has been developed within the Programme of Research and Technological Development from the Electricity Energy Sector regulated by ANEEL, with the support of the companies ENEVA - Pecém II Geração de Energia S.A., Iaqui Geração de Energia S. A., Parnaíba I, II and II Geração de Energia S. A.

\section{References}

1. Murakami, Y., Effect of Nonmetallic Inclusions of the Fatigue Strength. In Metal Fatigue: Effects of Small Defects and Nonmetallic Inclusions, p. 75-127. Oxford: Elsevier. (2002).

2. Yanase, K., \& Endo, M., Multiaxial high cycle fatigue threshold with small defects and cracks. Engineering Fracture Mechanics, p. 182-196. (2014).

3. Susmel, L., \& Lazzarin, P., A bi-parametric Wöhler Curve for High Cycle Multiaxial Fatigue Assesment. Fatigue \& Fracture of Engineerig Materials and Structures, p. 6378. (2002).

4. Endo, M., \& Ishimoto, I., The fatigue strength of steels containing small holes under out-of-phase combined loading. International Journal of Fatigue, p. 592-597. (2006).

5. Nadot, Y., \& Billaudeau, T., Multiaxial fatigue limit criterion for defective materials. Engineering Fracture Mechanics, p. 112-133. (2006).

6. Murakami, Y., Stress Concentration. Em Y. Murakami, Metal Fatigue: Effects of Small Defects and Nonmetallic Inclusions, Elsevier p. 28-41. (2002).

7. Jan Beirlant, Y. G., The probability side of extreme value theory. In Y. G. Jan Beirlant, Statistics of Extremes Theory and Applications, p. 45-80, John Wiley \& Sons, Ltd. (2004).

8. Araújo, J. A., Dantas, A. P., Castro, F. C., \& Mamiya, E. N. On the characterization of the critical plane with a simple and fast alternative measure of the shear stress ambplitude in multiaxial fatigue. International Journal of Fatigue, 1092-1100. (2011).

9. ASTM International, E466-15, Standard Practice for Controlled Constant Amplitude Axial Tests of Metallic Materials (2015).

10. International Council on Combustion Engines, IACS UR M53, Appendix IV. Guidance for Evaluiation of Fatigue Tests, (2009).

11. Murakami, Y., Appendix A: Instroctions for a New Method of Inclusion Rating and Correlations with the Fatigue Limit. In Y. Murakami, Metal Fatigue, Elsevier (2002). 